\title{
Expanding the spectrum of IDH1 mutations in gliomas
}

Ruta Gupta ${ }^{1,2}$, Simon Flanagan ${ }^{1,2}$, Cheryl CY Li ${ }^{3}$, Maggie Lee ${ }^{1,2}$, Brindha Shivalingham ${ }^{4}$, Sanaz Maleki ${ }^{2}$, Helen R Wheeler ${ }^{5}$ and Michael E Buckland ${ }^{1,2}$

${ }^{1}$ Department of Neuropathology, Royal Prince Alfred Hospital, Sydney, NSW, Australia; ${ }^{2}$ Discipline of Pathology, Sydney Medical School, University of Sydney, Sydney, NSW, Australia; ${ }^{3}$ Victor Chang Cardiac Research Institute, Sydney, NSW, Australia; ${ }^{4}$ Department of Neurosurgery, Royal Prince Alfred Hospital, Sydney, NSW, Australia and ${ }^{5}$ Department of Medical Oncology, Royal North Shore Hospital, St Leonards, NSW, Australia

\begin{abstract}
Mutations in isocitrate dehydrogenase -1 or -2 (IDH1 or IDH2) are found in the majority of WHO grade II and III diffuse gliomas and secondary glioblastomas. IDH mutation screening is rapidly becoming part of the routine pathological work up of human brain tumors, providing both diagnostic and prognostic information. Here, we characterize four rare and novel IDH1 mutations identified in surgical human glioma samples: two instances of an IDH1 p.R132S mutation caused by a previously undescribed dinucleotide deletion/insertion mutation, a novel homozygous somatic IDH1 p.R132L mutation, and an IDH1 p.R100Q mutation. Characterization of novel and rare IDH mutations may provide additional insight into the mechanisms of mutant IDH in neoplasia. Furthermore, given the clinical import of IDH status, these results highlight the need for comprehensive mutation screening, beyond the targeted identification of common pathogenic variants.
\end{abstract}

Modern Pathology (2013) 26, 619-625; doi:10.1038/modpathol.2012.210; published online 11 January 2013

Keywords: brain tumor; glioma; IDH; isocitrate dehydrogenase; mutation testing

IDH1 and IDH2 mutations were first identified during exome-wide sequencing of glioblastomas in $2008,{ }^{1}$ occurring predominantly in those that had arisen through progression of a lower grade glioma (ie, secondary glioblastomas). Subsequently, IDH mutations have been described in $70-80 \%$ of grade II and III astrocytomas, oligodendrogliomas and secondary glioblastomas, but are rare or nonexistent in other brain tumors. ${ }^{2}$ IDH mutations tend to occur in younger individuals and carry significant prognostic significance, in that those with $I D H-$ mutant tumors have longer disease-free survival and show better response to treatment. ${ }^{3-5}$ For these reasons, assessment of $I D H$ mutation status has rapidly become incorporated as a standard component in the neuropathological assessment of brain tumors. ${ }^{6}$

$I D H$ mutations in gliomas are largely known to be heterozygous missense mutations in codon R132 of $I D H 1$, or much less commonly, the synonymous codon (R172) of IDH2. These mutations result in

Correspondence: Dr ME Buckland, MD, PhD, FRCPA, Department of Neuropathology, University of Sydney and RPA Hospital, Room 502A, D06 Blackburn Building, Sydney, NSW 2006, Australia.

E-mail: michael.buckland@sydney.edu.au

Received 29 August 2012; revised 9 October 2012; accepted 9

October 2012; published online 11 January 2013 substitution of a highly conserved and structurally important arginine residue in the IDH substrate binding site. ${ }^{2,7}$ The most common IDH mutation is IDH1 p.R132H, accounting for around 90\% of IDHmutant glioma cases; less commonly, the IDH1 R132 substitution is with serine (p.R132S), leucine (p.R132L), glycine (p.R132G), cysteine (p.R132C) or valine (p. R132V) 2,8 and, at the molecular level, all but one of the reported cases are due to singlenucleotide substitutions. Mutations of IDH2 are relatively rare, accounting for $\sim 3-5 \%$ of all described $I D H$ mutations, but they carry similar prognostic implications. ${ }^{1}$

Mutations in $I D H$ genes are thought to contribute to neoplasia through a dominant-negative effect on the protein's wild-type function and/or a neomorphic gain of function of the mutant protein. ${ }^{6}$ Downstream effects of mutant IDH include decreased cellular NADPH and alpha-ketoglutarate $(\alpha \mathrm{KG})$ levels, ${ }^{9}$ HIF $1 \alpha$ stabilization, ${ }^{9}$ and increased production of 2-hydroxyglutarate (2HG), ${ }^{10}$ which competitively inhibits $\alpha$ KG-dependant enzymes such as histone methyltransferases and 5-methylcytosine hydroxylases. ${ }^{11}$ Homozygous mutations of IDH1 or IDH2 in gliomas have not been described, arguing against a simple loss of function mechanism. 
The focused nature of $I D H$ mutations has lent itself to a variety of detection techniques. Immunohistochemistry is a well-established component of diagnostic pathology workflows, and antibodies against the common IDH1 p.R132H mutant protein, as well as against a variant mutant protein, IDH1 p.R132S, are commercially available. ${ }^{12,13}$ Although the gold standard of clinical mutation detection is unbiased Sanger sequencing, other more focused methods such as pyrosequencing, ${ }^{14,15}$ PCR-based restriction fragment length polymorphism, ${ }^{16}$ highresolution melt (HRM) analysis, ${ }^{17,18}$ and single-base extension ${ }^{19}$ methods are commonly used. Most of these alternate mutation detection strategies require prior knowledge of the spectrum of possible mutations, and thus have the potential to miss novel variants.

Here, we report the identification and characterization of very rare and novel IDH1 mutations, encountered during clinical mutation assessment of human neurosurgical specimens. These findings expand the known spectrum of IDH mutations in human gliomas, and have important implications for the design of future mutation detection strategies.

\section{Materials and methods}

\section{Tumor Samples}

In all, 177 formalin-fixed paraffin-embedded (FFPE) brain tumors referred to the Royal Prince Alfred Hospital, Sydney, Australia for primary or secondary consultation were assessed by immunohistochemistry and bidirectional Sanger sequencing of exon 4 of IDH1 and IDH2; selected mutation cases were also interrogated by single-nucleotide primer extension (SNuPE) and/or pyrosequencing. Demographic and clinical details were obtained during routine diagnostic work up or referral notes. Tumor details are listed in Table 1.

\section{Immunohistochemistry}

Monoclonal antibodies against IDH1 p.R132H (clone H09; Dianova, Germany) and IDH1 p.R132S; (kind gift from Dr Y Kato, Japan) were used at 1:500 dilution on $5 \mu \mathrm{m}$-FFPE tumor sections, with antigen retrieval performed in $10 \mathrm{mM}$ sodium citrate buffer $\mathrm{pH}$ 6.0, for $20 \mathrm{~min}$ at $125^{\circ} \mathrm{C}$. Sections were incubated in primary antibodies for $1 \mathrm{~h}$ at room temperature and antibody detection was performed via Dako EnVision + (Dako, Carpinteria, USA) as per the manufacturer's instructions. Analysis for $1 \mathrm{p} / 19 \mathrm{q}$ codeletion was performed via fluorescence in situ hybridization (FISH) using commercially available labeled BAC probesets (Abbott-Vysis, USA) as per the manufacturer's recommendations and scored as previously described. ${ }^{20}$
Table 1 Tumors tested and IDH mutation status

\begin{tabular}{|c|c|c|c|c|}
\hline Diagnosis & $\begin{array}{l}\text { IDH } \\
\text { mutation }\end{array}$ & $\begin{array}{c}\text { IDH1 } \\
(\mathrm{R} 132 \mathrm{H})\end{array}$ & $\begin{array}{l}\text { IDH1 } \\
\text { (other) }\end{array}$ & $\begin{array}{c}\text { IDH2 } \\
\text { (R172K) }\end{array}$ \\
\hline $\begin{array}{l}\text { Astrocytoma (II), } \\
n=24\end{array}$ & $19(79 \%)$ & 13 & $\begin{array}{l}2 \text { (R132S) } \\
2 \text { (R132G) } \\
1 \text { (R132C) }\end{array}$ & 1 \\
\hline $\begin{array}{l}\text { Oligodendroglioma } \\
\text { (II), } n=14\end{array}$ & $14(100 \%)$ & 13 & 1 (R132S) & 0 \\
\hline Oligoastro (II), $n=5$ & $3(60 \%)$ & 3 & 0 & 0 \\
\hline $\begin{array}{l}\text { Anaplastic astro (III), } \\
n=35\end{array}$ & $14(40 \%)^{\mathrm{a}}$ & 8 & $\begin{array}{l}2 \text { (R132S) }^{\mathrm{b}} \\
3 \text { (R132G) }^{\mathrm{R} 132 \mathrm{~L}} \\
1 \text { (R132) }^{\mathrm{b}}\end{array}$ & 0 \\
\hline $\begin{array}{l}\text { Anaplastic oligo (III), } \\
n=7\end{array}$ & $7(100 \%)$ & 6 & 0 & 1 \\
\hline GBM (IV), $n=77$ & $15(19 \%)$ & 13 & $\begin{array}{l}1 \text { (R132S) }^{\mathrm{b}} \\
1 \text { (R100Q) }^{\mathrm{b}}\end{array}$ & 0 \\
\hline Other, $n=15$ & 0 & 0 & 0 & 0 \\
\hline
\end{tabular}

Abbreviation: IDH, isocitrate dehydrogenase.

Other tumors tested (bottom row) consisted of gliosarcoma (2), ependymoma (2), anaplastic ependymoma (1), pilocytic astrocytoma (4), central neurocytoma (2), subependymal giant cell astrocytoma (1), ganglioglioma (1), rosette forming glioneuronal tumor (1), and dysembryoplastic neuroepithelial tumor (1).

aThe anaplastic astrocytoma category included a group of referred H09-immunonegative tumors, biasing the proportion of mutated tumors in this subgroup.

${ }^{\mathrm{b}}$ Denotes the rare and novel mutations reported in this manuscript.

\section{DNA Extraction and PCR}

DNA extraction of each sample was performed on five $10 \mu \mathrm{m}$ sections of FFPE tissue, which were deparaffinized by washing with xylene twice, followed by a single ethanol wash. Extraction from the deparaffinized tissue was performed using the Qiagen DNEasy blood and tissue kit (Qiagen, Hilden, Germany), according to the manufacturer's protocol. All PCRs were performed in $50 \mathrm{mM}$ Tris/ $\mathrm{HCl}, 10 \mathrm{mM} \mathrm{KCl}, 5 \mathrm{mM}\left(\mathrm{NH}_{4}\right)_{2} \mathrm{SO}_{4}$ and $2 \mathrm{mM}$ $\mathrm{MgCl}_{2}$, with $0.2 \mathrm{mM}$ of each dNTP, 2U of FastStart Taq polymerase (Roche, Manheim, Germany), $0.5 \mu \mathrm{M}$ of each primer and $200 \mathrm{ng}$ DNA in a total volume of $20 \mu \mathrm{l}$. PCR cycling conditions consisted of initial denaturation at $95{ }^{\circ} \mathrm{C}$ for $5 \mathrm{~min}$, followed by 40 cycles of denaturation, annealing and extension at $95{ }^{\circ} \mathrm{C}$ for $30 \mathrm{~s}, 60{ }^{\circ} \mathrm{C}$ for $30 \mathrm{~s}$, and $72{ }^{\circ} \mathrm{C}$ for $30 \mathrm{~s}$, respectively.

\section{Sequencing}

Bidirectional Sanger sequencing of exon 4 of IDH1 and exon 4 of $I D H 2$ was performed on PCR products generated using the primer pairs IDH1e4_1F/ IDH1e4_2RS and IDH2e4F/IDH2e4R, respectively, (see Table 2 for primer sequences). PCR products were sequenced bidirectionally using M13-F and M13-R primers using BigDye Terminator v3.1 Cycle Sequencing Kit (Applied Biosystems), according to the manufacturer's instructions. Sequencing 
reaction products were resolved on a $3730 \times 1$ DNA Analyzer (Applied Biosystems).

Pyrosequencing was performed on a 155-bp fragment spanning codon 132 of IDH1, amplified using primers IDH1_pyroF and IDH1_pyroR (see Table 2 for primer sequences). Pyrosequencing of PCR products was performed using the Pyromark Q24 pyroseqencing platform (Qiagen, Hilden, Germany) using the internal sequencing primer IDH1_pyroS (Table 2) according to the manufacturer's instructions.

\section{SNuPE and Loss of Heterozygosity (LOH) Analysis}

SNuPE was performed on two microlitres of the $155 \mathrm{bp}$ PCR product from the pyrosequencing assay. It was analyzed using the Snapshot Multiplex System (Applied Biosystems) and interrogation primers IDH1_INT1 and IND1_INT2 (Table 2), according to the manufacturer's instructions.

LOH analysis was assessed by PCR amplification of the microsatellite markers D2S325, D2S2321, D2S1276, D2S2178 and D2S2274 using fluorescently labeled primers. Primer sequences for each marker were retrieved from NCBI's UniSTS database (http://www.ncbi.nlm.nih.gov/sites/entrez?db=unists). PCRs were performed as described above, but with only 30 cycles of denaturing, annealing and extension at $95{ }^{\circ} \mathrm{C}$ for $30 \mathrm{~s}, 55^{\circ} \mathrm{C}$ for $30 \mathrm{~s}$ and $72{ }^{\circ} \mathrm{C}$ for $30 \mathrm{~s}$, respectively. Products were resolved on a 3730xl DNA Analyzer (Applied Biosystems).

\section{Results}

\section{IDH Mutations in an Australian Glioma Cohort}

The 177 brain tumors in this study were from 77 males and 100 females. The mean age was 54 years (median age 55.5 years), and there was no

Table 2 PCR, sequencing and SNuPE primers used in the study

\begin{tabular}{ll}
\hline Name & Sequence $5^{\prime}-3^{\prime}$ \\
\hline M13-F & TGTAAAACGACGGCCAGT \\
M13-R & CAGGAAACAGCTATGACC \\
IDH1e4_1F & TGTAAAACGACGGCCAGT \\
& catttgtctgaaaactttgctt \\
IDH1e4_2RS & CAGGAAACAGCTATGACC \\
& tcacattattgccaacatgac \\
IDH1_pyroF & AAATCACCAAATGGCACCATA \\
IDH1_pyroR & TTGCCAACATGACTTACTTGATC \\
IDH1_pyroS & GACTTACTTGATCCCCATA \\
IDH1_INT1 & GACTGACTGATGGGTAAAACT \\
& TATCATCATAGGT \\
IDH1_INT2 & ACTGACTTTGATCCCCATAAGCATGA \\
IDH2 e4 F & TGTAAAACGACGGCCAGT \\
IDH2 e4 R & ggttcaaattctggttgaaagatg \\
& CAGGAAACAGCTATGACC \\
& gctaggcgaggagctccagt
\end{tabular}

Abbreviation: IDH, isocitrate dehydrogenase; SNuPE, single-nucleotide primer extension. gender difference in age of onset. The tumor type and mutation status is summarized in Table 1. A number of anaplastic astrocytomas included in this cohort were previously identified as IDH1 (p.R132H)-immunonegative by another laboratory and were referred to us for sequencing; thus, there is likely ascertainment bias accounting for the lower than expected proportion of $I D H$-mutant tumors in this subgroup. Overall, the clinicopathological features of our glioma cohort are similar to those described from other centers across the world. However, four of the 72 mutations we identified were very unusual or completely novel, and are described in detail below.

\section{Two Cases of a Novel Dinucleotide Deletion/Insertion Mutation Leading to IDH1 p.R132S}

Two gliomas, an anaplastic astrocytoma and a glioblastoma, were found to contain a heterozygous dinucleotide deletion/insertion at IDH1 codon 132 (c.394_395CGdelinsTC, p.R132S) on Sanger sequencing (Figure 1). Sequencing was unable to discriminate between involvement of a single IDH1 allele with the dinucleotide deletion/insertion or a compound heterozygous mutation involving both IDH1 alleles (Figure 1a). Immunohistochemistry using the IDH1 p.R132S monoclonal antibody SMab-1 demonstrated strong immunoreactivity of tumor cells in the anaplastic astrocytoma, confirming the presence R132S mutant protein expression (Figure 1b) and hence the dinucleotide deletion/ insertion event. The glioblastoma was R132S-immunonegative. Multiplex IDH1 SNuPE assay confirmed the presence of mutations at both c.394 and c.395 positions (Figure 1c), but we cannot exclude a compound heterozygous mutation in this tumor. The R132S-immunopositive anaplastic astrocytoma was removed from a 59-year-old male with a nonenhancing temporoparietal tumor who had previously had a WHO grade II astrocytoma removed 9 years earlier without adjuvant therapy. He is alive and disease free 38 months post resection. His initial resection was unavailable for testing. The immunonegative glioblastoma arose in the splenium of a 67-year-old female, without clinical, radiological or pathological evidence of pre-existing low-grade glioma.

\section{An IDH1 p.R132L Mutation Associated with LOH of IDH1}

A 5-cm well-circumscribed, solid, non-enhancing lesion in the left parietal lobe was discovered incidentally in a 36-year-old female (Figure 2a). She underwent gross total resection of the tumor, and neuropathological examination indicated an anaplastic astrocytoma, WHO grade III (Figure 2b). There was no 1p36.3 or 19q.13 loss (as determined by FISH), and IDH1 p.R132H immunohistochemistry 
a
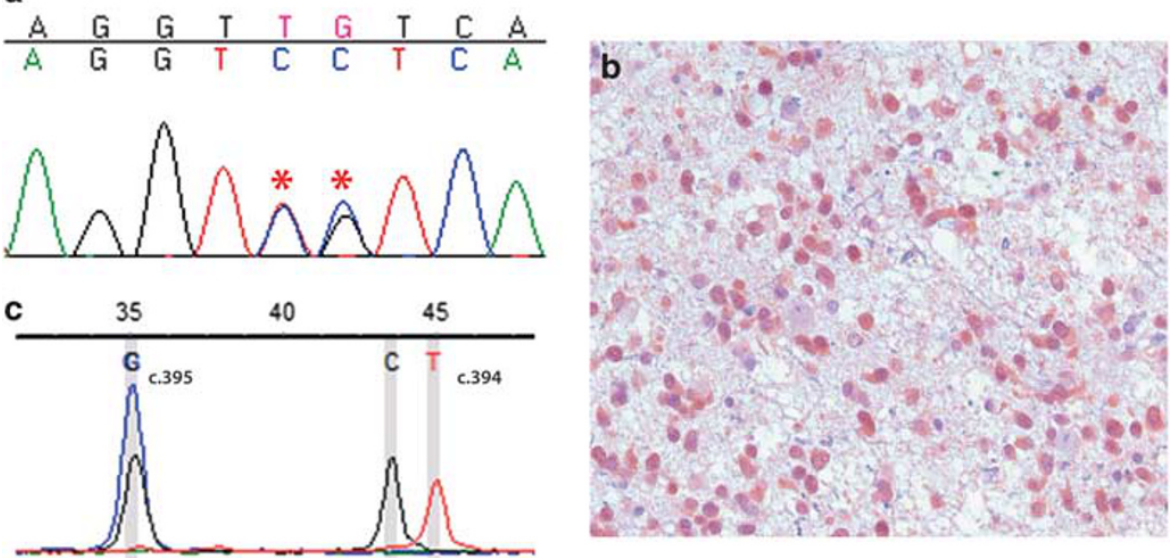

Figure 1 A two base pair insertion/deletion causing IDH1 p.R132S. (a) Sanger sequencing of DNA extracted from an anaplastic astrocytoma demonstrated heterozygous dinucleotide deletion/insertion of IDH1 codon 132 (c.394_395CGdelinsTC, p.R132S; asterisks (*)). Sanger sequencing was unable to discriminate between involvement of a single IDH1 allele with the dinucleotide mutation and a compound heterozygous mutation involving both IDH1 alleles. (b) Immunohistochemistry with SMab-1 confirmed R132S mutant protein expression restricted to tumor cells. (Original magnification: $\times 400$ ). (c) Multiplex IDH1 SNuPE confirmed the Sanger sequencing of an additional case of dinucleotide insertion/deletion mutation in a glioblastoma, which was immunonegative for SMab-1.

was negative. Bidirectional Sanger sequencing demonstrated a seemingly homozygous IDH1 p.R132L mutation (Figure 2c). Quantitative pyrosequencing of codon 132 of $I D H 1$ indicated the presence of $76 \%$ mutant allele and 24\% wild-type allele (Figure 2d). There was no family history of brain tumors or other malignancies, and the patient's peripheral blood was normal (not shown), militating against a germline defect. $\mathrm{LOH}$ analysis for the microsatellite markers D2S322, D2S2274, D2S2208, D2S2242 and D2S2321 flanking the IDH1 locus on $2 \mathrm{q}$ was performed on the patient's tumor and peripheral blood. This demonstrated $\mathrm{LOH}$ of all three informative markers (D2S2321, D2S2178, D2S2274) restricted to tumor DNA (Figure 2e). D2S2321 is centromeric while D2S2178 and D2S2274 are telomeric to IDH1, confirming $\mathrm{LOH}$ of at least $2 \mathrm{Mb}$ encompassing the entire IDH1 gene, with retention of the mutant allele in tumor DNA. The patient had postoperative adjuvant therapy (radiation and temozolomide) and is disease-free at 18 months post resection.

\section{IDH1 p. R100Q Mutation}

A 54-year-old female patient had an enhancing left insular tumor. Formal histopathology revealed a mixed oligoastrocytic tumor with focal necrosis. In the previous WHO classification (2000), this tumor would be classified as a WHO grade III anaplastic oligoastrocytoma, however, the presence of focal necrosis in the specimen now qualifies it as a glioblastoma with oligodendroglioma component, WHO (2007) grade IV $^{21}$ (Figure 3a). FISH revealed an isolated 19q deletion. IDH1 p.R132H immunohistochemistry on the tumor was negative. Codon 132 of IDH1 and codon 172 of IDH2 were wild-type on bidirectional Sanger sequencing. However, the
IDH1 sequencing amplicon also included codon R100, and identified a heterozygous IDH1 p. R100Q mutation (c.299G > A, p.R100Q) (Figure 3b). Unfortunately, this patient was from a distant regional center and was lost to follow-up 6 months postdischarge, so long term outcome information was not available.

\section{Discussion}

The relative frequency and clinical characteristics of IDH mutated gliomas in this Australian cohort are similar to those described by in reports from a variety of centers across the globe including the United States, ${ }^{2}$ India,${ }^{22}$ Germany, ${ }^{8}$ France,${ }^{5}$ China ${ }^{23}$ and Japan. ${ }^{24}$ However, a striking finding is the frequency of rare or previously undescribed mutations in our cohort. This may in part be explained by the fact that many laboratories rely on more focused mutation detection methods such as immunohistochemistry, HRM or singlenucleotide extension assays, which are designed to detect known/characterized $I D H$ mutations and may not recognize uncharacterised or rare variants. Australia has a diverse mix of ethnic groups, including a distinct indigenous population as well as numerous residents of South East Asian, Polynesian and Melanesian background, and it is possible that certain racial haplotypes may have a higher frequency of unusual mutations.

Dinucleotide deletion/insertion mutations, or tandem base pair mutations, are uncommon genetic lesions in both human somatic and germline disease. ${ }^{25}$ Only one dinucleotide deletion/insertion mutation of IDH1 has been reported previously in gliomas, in that case a (c.394_395CGdelinsGT, p.R132V) was identified in a study of 685 brain 

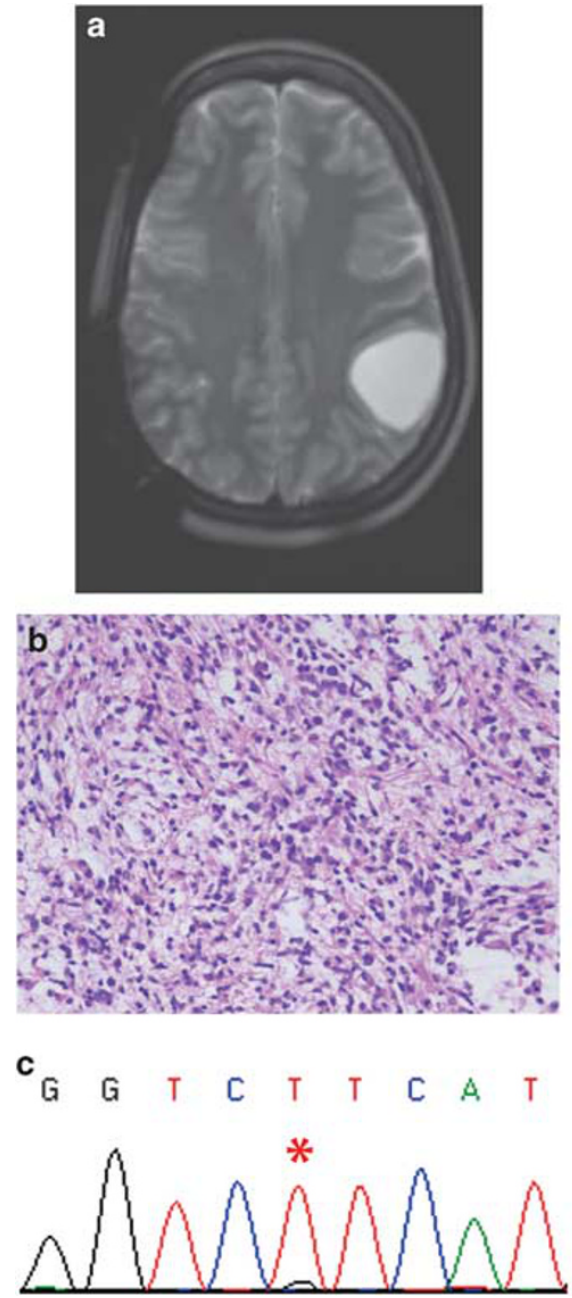

d

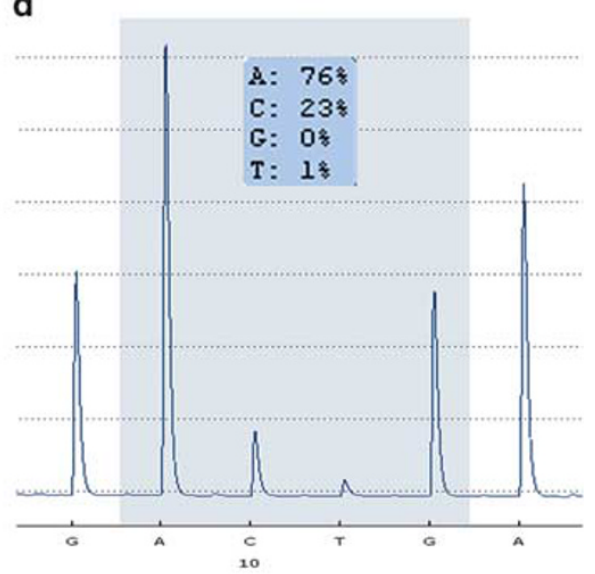

e

Tumour

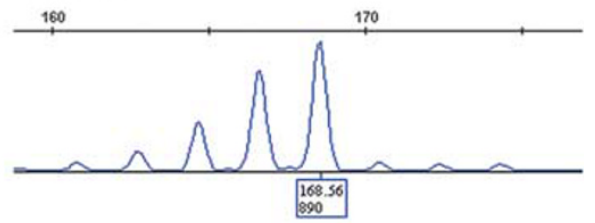

Peripheral blood

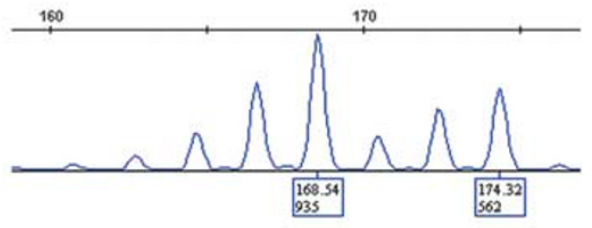

Figure 2 A novel IDH1 p.R132L mutation with loss of heterozygosity (LOH). (a) Axial T1-weighted MRI of a 5-cm well-circumscribed non-enhancing tumor in the left parietal lobe. (b) Haematoxylin and eosin (H\&E) stained sections confirmed an anaplastic astrocytoma WHO grade III (original magnification: $\times 200$ ). (c) Sanger sequencing of IDH1 exon 4 demonstrates a seemingly homozygous IDH1 (c.395G > T, p.R132L) mutation (asterisk). (d) Quantitative pyrosequencing confirms the presence of $76 \%$ mutant allele and $24 \%$ wildtype allele, consistent with homozygous mutant tumor admixed with some non-neoplastic cells. (e) LOH analysis for the microsatellite markers D2S322 (shown), D2S2274, D2S2208, D2S2242 and D2S2321 flanking the IDH1 locus on 2q on the patients tumor and peripheral blood demonstrated LOH of all three informative markers in tumor DNA, confirming LOH of at least 2 Mb encompassing the entire IDH1 gene, with retention of the homozygous mutant allele in tumor DNA.

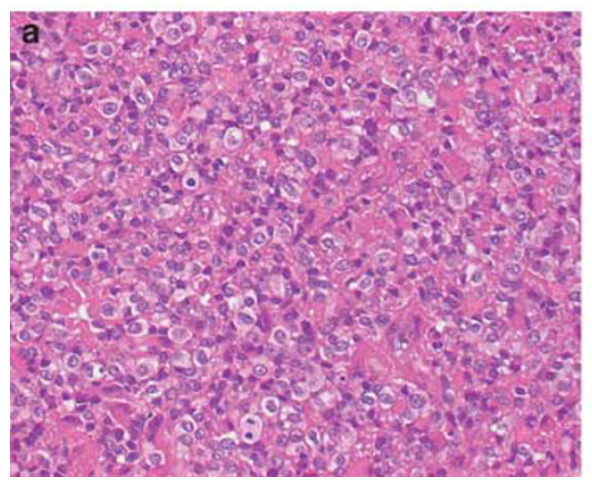

b

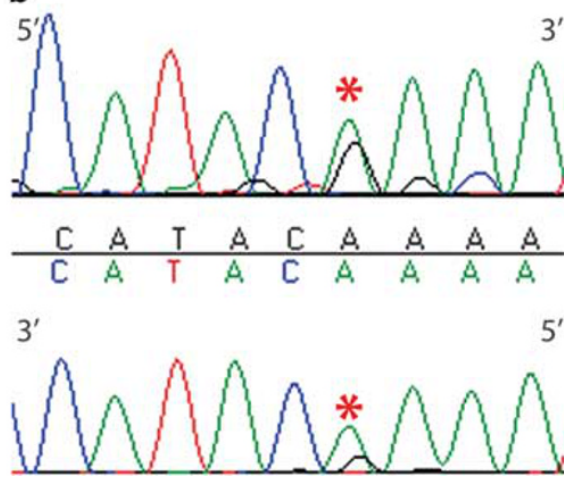

Figure 3 (a) A glioblastoma with oligodendroglioma component, WHO (2007) grade IV. (H\&E stain, original magnification: $\times 200$ ). (b) A heterozygous missense IDH1 p.R100Q mutation (c.299G > A, p.R100Q) was identified by bidirectional Sanger sequencing. No other IDH1 or IDH2 exon 4 mutations were present. 
tumors, ${ }^{8}$ the only reported example of the p.R132V mutation. These unusual dinucleotide mutations have also been seen as rare events in AML patients, and four instances of heterozygous dinucleotide deletion/ insertion mutation of IDH1 (c.395_396GTdelinsAC) as an alternate path to the IDH1 p.R132H mutant protein have been reported in a study of 416 Chinese AML patients. ${ }^{26}$ Ultraviolet radiation and cis-platinum chemotherapy are commonly associated with these dinucleotide deletion/insertion mutations, ${ }^{27}$ but neither of these factors appear relevant to the two cases described above. The fact that these unusual mutations in both gliomas and AML are missense mutations involving the critical arginine residue at codon 132 is further evidence of the importance of this residue in the effects of mutant IDH1 in neoplasia.

This cohort also includes an apparently homozygous IDH1 p.R132L mutation due to $\mathrm{LOH}$ at the IDH1 allele. The pathological features of this case were of a typical anaplastic astrocytoma, although the clinical presentation (an incidental finding) and radiological characteristics were slightly unusual. To our knowledge, only a single case of partial $\mathrm{LOH}$ of mutant $I D H 1$ has been previously characterized. In that case, $\mathrm{LOH}$ was identified on progression of a diffuse astrocytoma to secondary glioblastoma with adjuvant radiotherapy. ${ }^{28}$ However, in contrast to the current case, this previous case demonstrated partial loss of the mutant IDH1 allele, rather than a loss of the wild-type IDH1 allele described here. Furthermore, the current case was a primary (ie, untreated) tumor, with homozygous IDH1 mutation status identified on initial testing. Although $\mathrm{LOH}$ in neoplasia is most typically associated with tumor suppressor genes, where it contributes to loss of function of that gene, in vitro evidence indicates that mutant IDH1 is capable of $2 \mathrm{HG}$ production, both in the absence and presence of expression of the wildtype IDH1 allele. ${ }^{10}$ In fact, co-expression of wildtype IDH1 with the mutant protein (as is the case in most IDH1-mutated gliomas) may enhance 2HG production, as wild-type IDH1 activity increases levels of NADPH and $\alpha$ KG, both necessary substrates for neomorphic production of $2 \mathrm{HG} \cdot{ }^{10} \mathrm{LOH}$ of the $I D H 1$ locus is very uncommon in gliomas, ${ }^{29}$ while homozygous IDH1 mutations in AML are very rare, ${ }^{26}$ and whether the clinical behavior of these tumors is distinct from those with typical $I D H$ mutations is not known.

In addition to R132, there are two other arginines present in the IDH1-substrate binding site. The IDH1 R100 residue is synonymous with the IDH2 R140 residue, which is commonly mutated in myeloid malignancies. In vitro assessment of the IDH1 p. R100Q mutant has demonstrated that it results in overproduction of 2-hydroxy glutarate $(2 \mathrm{HG})^{30}$ in a comparable manner to IDH1 p.R132 and IDH2 p.R140 mutations. The case reported here represents the fourth known example of the IDH1 p. R100Q mutation in gliomas; the three other instances described previously in a single report. ${ }^{28}$
The prevalence of mutations in the synonymous IDH2 arginine residue (R140) in myeloid malignancies and the similar functional consequences of both the IDH1 p.R132H and p.R100Q mutations upon 2HG levels ${ }^{30}$ suggests that this mutation would have similar clinical implications to the typical IDH1 R132 mutations.

These results indicate that the spectrum of $I D H 1$ defects in adult gliomas is more diverse than would be expected from the current literature, and that direct sequencing of exon 4 of IDH1 and 2 has the potential to identify non-canonical mutations, which may be missed by other methods.

\section{Note added in proof}

Between acceptance and publication of this manuscript, Jin and colleagues ${ }^{31}$ have reported two additional cases of $\mathrm{LOH}$ at the $I D H 1$ allele in gliomas. In comparison to our case of de novo $\mathrm{LOH}$, these additional cases were identified upon progression of IDH-mutant anaplastic astrocytomas to secondary glioblastomas.

\section{Acknowledgements}

The authors thank Roger Stankovic, Tina Selinger and Trina Lum for technical assistance, and Catherine Suter for critical reading of the manuscript. MEB is a Cancer Institute NSW Clinical Research Fellow.

\section{Disclosure/conflict of interest}

Dr Buckland is a Cancer Institute NSW Clinical Research Fellow. The remaining authors declare no conflict of interest.

\section{References}

1 Parsons DW, Jones S, Zhang X, et al. An integrated genomic analysis of human glioblastoma multiforme. Science 2008;321:1807-1812.

2 Yan H, Parsons DW, Jin G, et al. IDH1 and IDH2 mutations in gliomas. N Engl J Med 2009;360:765-773.

3 Metellus P, Coulibaly B, Colin C, et al. Absence of IDH mutation identifies a novel radiologic and molecular subtype of WHO grade II gliomas with dismal prognosis. Acta Neuropathol 2011;120:719-729.

4 Hartmann C, Hentschel B, Wick W, et al. Patients with IDH1 wild-type anaplastic astrocytomas exhibit worse prognosis than IDH1-mutated glioblastomas, and IDH1 mutation status accounts for the unfavorable prognostic effect of higher age: implications for classification of gliomas. Acta Neuropathol 2010;120:707-718.

5 Houillier C, Wang X, Kaloshi G, et al. IDH1 or IDH2 mutations predict longer survival and response to temozolomide in low-grade gliomas. Neurology 2010;75:1560-1566.

6 Gupta R, Webb-Myers R, Flanagan S, et al. Isocitrate dehydrogenase mutations in diffuse gliomas: clinical 
and aetiological implications. J Clin Pathol 2011;64:835-844.

7 Reitman ZJ, Parsons DW, Yan H. IDH1 and IDH2: not your typical oncogenes. Cancer Cell 2010;17: 215-216.

8 Balss J, Meyer J, Mueller W, et al. Analysis of the IDH1 codon 132 mutation in brain tumors. Acta Neuropathol 2008;116:597-602.

9 Zhao S, Lin Y, Xu W, et al. Glioma-derived mutations in IDH1 dominantly inhibit IDH1 catalytic activity and induce HIF-1alpha. Science 2009;324:261-265.

10 Dang L, White DW, Gross S, et al. Cancer-associated IDH1 mutations produce 2-hydroxyglutarate. Nature 2009;462:739-744.

$11 \mathrm{Xu} \mathrm{W}$, Yang H, Liu Y, et al. Oncometabolite 2-hydroxyglutarate is a competitive inhibitor of alpha-ketoglutarate-dependent dioxygenases. Cancer Cell 2011;19:17-30.

12 Capper D, Zentgraf H, Balss J, et al. Monoclonal antibody specific for IDH1 R132H mutation. Acta Neuropathol 2009;118:599-601.

13 Kato Y, Jin G, Kuan CT, et al. A monoclonal antibody IMab-1 specifically recognizes IDH1R132H, the most common glioma-derived mutation. Biochem Biophys Res Commun 2009;390:547-551.

14 Felsberg J, Wolter M, Seul H, et al. Rapid and sensitive assessment of the IDH1 and IDH2 mutation status in cerebral gliomas based on DNA pyrosequencing. Acta Neuropathol 2010;119:501-507.

15 Setty P, Hammes J, Rothamel T, et al. A pyrosequencing-based assay for the rapid detection of IDH1 mutations in clinical samples. J Mol Diagn 2010;12:750-756.

16 Bujko M, Kober P, Matyja E, et al. Prognostic Value of IDH1 Mutations Identified with PCR-RFLP Assay in Glioblastoma Patients. Mol Diagn Ther 2010;14: 163-169.

17 Patel KP, Barkoh BA, Chen Z, et al. Diagnostic testing for IDH1 and IDH2 variants in acute myeloid leukemia an algorithmic approach using high-resolution melting curve analysis. J Mol Diagn 2011;13:678-686.

18 Horbinski C, Kelly L, Nikiforov YE, et al. Detection of IDH1 and IDH2 Mutations by Fluorescence Melting Curve Analysis as a Diagnostic Tool for Brain Biopsies. J Mol Diag 2010;12:487-492.

19 Perizzolo M, Winkfein B, Hui S, et al. IDH mutation detection in formalin-fixed paraffin-embedded gliomas using multiplex PCR and single-base extension. Brain Pathol 2012;22:619-624.

20 Smith JS, Perry A, Borell TJ, et al. Alterations of chromosome arms $1 p$ and $19 q$ as predictors of survival in oligodendrogliomas, astrocytomas, and mixed oligoastrocytomas. J Clin Oncol 2000;18:636-645.

21 Louis DN, Ohgaki H, Wiestler OD, Cavenee WK (eds) WHO Classification of Tumours of the Central Nervous System. IARC: Lyon, 2007;309 pp.

22 Jha P, Suri V, Sharma V, et al. IDH1 mutations in gliomas: first series from a tertiary care centre in India with comprehensive review of literature. Exp Mol Pathol 2011;91:385-393.

23 Qi ST, Yu L, Lu YT, et al. IDH mutations occur frequently in Chinese glioma patients and predict longer survival but not response to concomitant chemoradiotherapy in anaplastic gliomas. Oncol Rep 2011;26:1479-1485.

24 Mukasa A, Takayanagi S, Saito K, et al. Significance of IDH mutations varies with tumor histology, grade, and genetics in Japanese glioma patients. Cancer Sci 2012;103:587-592.

25 Burkitt-Wright EM, Bradley L, Shorto J, et al. Neonatal lethal Costello syndrome and unusual dinucleotide deletion/insertion mutations in HRAS predicting p.Gly12Val. Am J Med Genet A 2012;158A:1102-1110.

26 Zhang Y, Wei H, Wang M, et al. Some novel features of IDH1-mutated acute myeloid leukemia revealed in Chinese patients. Leuk Res 2011;35:1301-1306.

27 Hutchinson F. Induction of tandem-base change mutations. Mutat Res 1994;309:11-15.

28 Pusch S, Sahm F, Meyer J, et al. Glioma IDH1 mutation patterns off the beaten track. Neuropathol Appl Neurobiol 2011;37:8.

29 Ichimura K, Pearson DM, Kocialkowski S, et al. IDH1 mutations are present in the majority of common adult gliomas but rare in primary glioblastomas. Neuro Oncol 2009;11:341-347.

30 Ward PS, Cross JR, Lu C, et al. Identification of additional IDH mutations associated with oncometabolite R(-)-2-hydroxyglutarate production. Oncogene 2012;31:2491-2498.

31 Jin G, Reitman ZJ, Duncan CG, et al. Disruption of wild type IDH1 suppresses D-2-hydroxyglutarate production in IDH1-mutated gliomas. Cancer Res; advance online publication, 30 November 2012 (e-pub ahead of print). 